\title{
COAGULOPATIA NO TRAUMA
}

\author{
COAGULOPATHY IN TRAUMA
}

Bartolomeu Nascimento Júnior ${ }^{1}$, Sandro Scarpelini², Sandro Rizoli ${ }^{3}$

\begin{abstract}
${ }^{1}$ Critical Care Fellow. University of Toronto. Department of Critical Care Medicine. Sunnybrook Health Sciences Centre. ${ }^{2}$ Docente. Disciplina de Cirurgia de Urgência e Trauma. Departamento de Cirurgia e Anatomia. Faculdade de Medicina de Ribeirão Preto - USP. ${ }^{3}$ Associated Professor of Surgery and Critical Care Medicine. University of Toronto. Department of Surgery and Critical Care Medicine. Sunnybrook Health Sciences Centre.

Correspondência: Sunnybrook Health Sciences Centre. 2075 Bayview Ave., Suíte H1-71 M4N 3M5, Toronto / Canadá. sandro.rizoli@sunnybrook.ca
\end{abstract}

Nascimento Jr. B, Scarpelini S, Rizoli S. Coagulopatia no trauma. Medicina (Ribeirão Preto) 2007; 40 (4): 509-17, out./dez.

RESUMO: Trauma é uma das principais causas de mortalidade no mundo. A principal causa de óbitos das vítimas de trauma, nas primeiras 24 horas após a lesão, é o sangramento maciço. Os pacientes politraumatizados podem desenvolver graves distúrbios de coagulação relacionados à perda de grandes volumes de sangue e fatores de coagulação, à reposição volêmica com cristalóides e com concentrados de hemácias, à acidose e à hipotermia. $\mathrm{O}$ controle deste tipo de situação tem sido um grande desafio para cirurgiões e intensivistas. Além disto, as vítimas de trauma formam um dos maiores grupos de consumidores de sangue e seus derivados. Recentemente, a adequação destes grandes volumes de transfusão tem sido questionada, principalmente em decorrência da ausência de protocolos bem definidos e testados clinicamente. $O$ objetivo deste artigo foi rever a literatura atual conceituando coagulopatia do trauma e descrevendo as mais recentes orientações para a hemoterapia direcionada às vitimas de trauma.

Descritores: Trauma. Sangramento Maciço. Coagulopatia. Transfusão Sangüínea.

\section{1- INTRODUÇÃO}

Atualmente, a morte decorrente de traumatismos é, sem duvida, um problema de saúde pública mundial. Estimativas internacionais indicam que, entre 5 e 44 anos de idade, o trauma é a primeira causa de morte sendo responsável por $10 \%$ de todos os óbitos. ${ }^{1}$ No Brasil, desde 1980, o trauma detém um lugar de liderança entre as principais causas de morte. ${ }^{2} \mathrm{Em}$ 2004, foram computadas cerca de 150 mil mortes decorrentes de traumatismos e as estimativas indicam cerca de 800.000 internações anuais, contabilizando apenas os hospitais públicos. ${ }^{3}$
Estudos epidemiológicos mostram que o sangramento é o grande responsável pelos óbitos ocorridos nas primeiras 24 horas após o trauma. ${ }^{4,5}$

As vítimas de trauma com lesões de maior gravidade podem apresentar sangramento maciço que, associado a alterações hematológicas induzidas pelo próprio tratamento realizado para restabelecer a perfusão adequada aos órgãos vitais, resulta em um distúrbio na coagulação. Na maioria das vezes, este distúrbio é de difícil controle e, quando não corrigido, leva o traumatizado inevitavelmente à morte.

Este artigo tem como objetivos proporcionar uma revisão sobre a fisiopatologia da coagulopatia 
que se desenvolve nos traumatizados graves, os recursos atuais disponíveis para o seu diagnóstico, assim como as dificuldades e limitações destes métodos, as estratégias para prevenção e tratamento deste distúrbio e perspectivas futuras na área.

\section{2- FISIOPATOLOGIA DA COAGULOPATIA DO TRAUMA}

Coagulopatia é a falência do sangue em produzir hemostasia adequada em resposta a uma lesão tecidual, seja esta decorrente de traumatismos, intervenções cirúrgicas ou de método diagnóstico invasivo. Esta falência é também conhecida como o "sangramento não-cirúrgico ou micro-vascular". Em condições normais de hemostasia este processo envolve, resumidamente, três etapas essenciais:

1- a ligação do fator VII ao fator tecidual, que é exposto durante a lesão vascular, originando o complexo "Fator tecidual/Fator VII ativado" que inicia a cascata da coagulação;

2- a ativação de plaquetas e fatores de coagulação com conseqüente produção de rede de fibrina (Figura 1); e

3- a ativação da via da fibrinólise que previne a formação de coagulação excessiva.
O desenvolvendo da coagulopatia do trauma é resultado da combinação de vários fatores associados (Tabela I).

Atualmente, a maioria dos grandes centros especializados no tratamento das vítimas de trauma adota as recomendações do "Advanced Trauma Life Support - ATLS ${ }^{\circledR ”}$ do “American College of Surgeons - ACS", como rotina de atendimento ${ }^{6}$. Baseado na classificação clínica da gravidade do choque hemorrágico, que varia desde as classes I e II de choque (Grau I até $15 \%$ e Grau II de 15 a 30\% da volemia total), onde o grau de sangramento geralmente é corrigido apenas com a reposição de cristalóides ( $2-4$ litros de ringer lactato ou soro fisiológico inicialmente), até as classes III e IV de choque (Grau III de 30 a $40 \%$ e Grau IV mais de $40 \%$ da volemia total), que requerem não só a reposição de fluidos, mas também a transfusão sangüínea. Nas classes mais graves de choque existem perdas significativas de componentes do sangue que, a partir de determinado ponto, resultam inevitavelmente no desenvolvimento de defeitos no processo normal de hemostasia. Na vigência de sangramento, este processo é ativado resultando no consumo de fatores de coagulação e plaquetas.

A própria reposição volêmica com fluidos e hemocomponentes pode conduzir a alterações na coa-

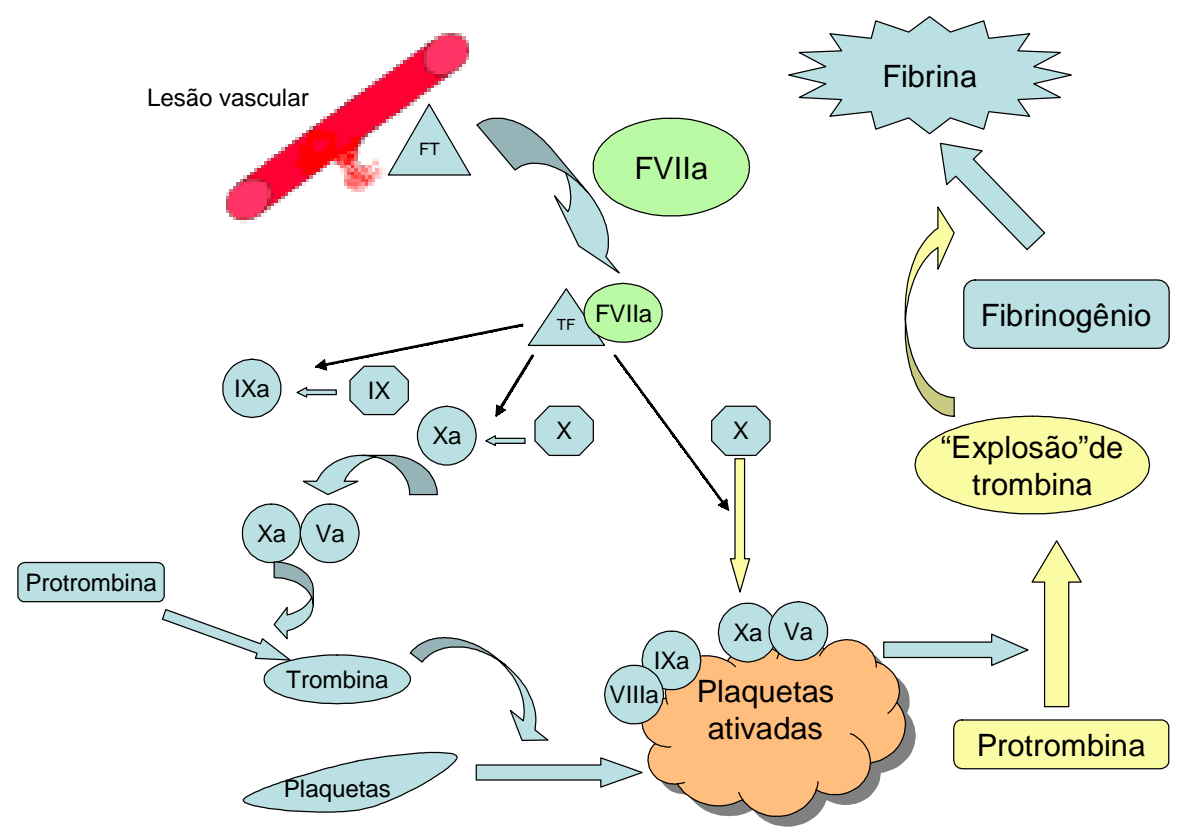

Figura 1: Descrição geral do processo de formação do coágulo e da ação do fator VII ativado.

$\mathrm{O}$ fator VII forma um complexo (FVIla-FT) com o fator tecidual exposto na lesão vascular. Este complexo ativa os fatores IX e X resultando em uma "explosão" de trombina e uma formação rápida da rede de fibrina no local da lesão.

(FT - Fator tecidual; Va - Fator V ativado; FVIla - Fator VII ativado; VIIla - Fator VIII ativada; IX - Fator IX; X - Fator X; Xa - Fator $X$ ativado). 
Tabela I: Fatores associados com o desenvolvimento da coagulopatia no trauma

Fatores etiológicos Conseqüências Hematológicas

Perda sanguínea

- Redução na quantidade absoluta de fatores de coagulação e plaquetas.

Ativação da Hemostasia

- Consumo de Plaquetas e fatores de coagulação.

Administração de fluidos

- Redução da quantidade relativa de fatores de coagulação e plaquetas.

- Reposição volêmica agressiva com soro fisiológico pode piorar a acidose devido ao desenvolvimento da acidose metabólica hiperclorêmica.

- Transfusão de concentrado de hemácias isoladamente leva a deficiência relativa de plaquetas e fatores de coagulação e também pode levar a hipocalcemia e hipotermia.

- Alguns estudos em animais sugerem que alguns colóides têm efeito direto na coagulação.

- Hipotermia com seus efeitos deletérios na hemostasia.

Acidose - Redução da atividade dos fatores de coagulação e seus complexos; principalmente o fator VII, complexo fator VII/fator tecidual e o complexo fator Xa/ $\mathrm{Va}$.

Hipotermia

- Redução da atividade das enzimas sereases envolvidas na função plaquetária e por meio de alterações endoteliais e no sistema fibrinolítico.

Traumatismo Craniano

- Acredita-se que a liberação de tromblopastina e fator tecidual decorrente de traumatismo craniano iniciam o processo de coagulação e fibrinólise similar à coagulação intravascular disseminada.

gulação, decorrentes da diluição dos elementos envolvidos neste processo. Na nossa experiência, cerca de 3 a $4 \%$ dos traumatizados desenvolvem sangramentos graves que requerem transfusão sanguínea maciça, isto é, transfusão de mais de 10 unidades de concentrado de hemácias dentro das primeiras 24 horas $^{7}$, com índices de mortalidade que alcançam $45 \% .8$

Com este nível de perda sanguínea e conseqüente reposição volêmica agressiva com fluidos, a maioria dos doentes desenvolverá acidose metabólica e hipotermia o que, no trauma, quando associado à coagulopatia forma a conhecida "Lethal Triad" (Triade Letal), que se não revertida ocasionara a morte do paciente.

Descrito desde a primeira guerra mundial, este ciclo de reposição agressiva de fluidos, seguido por sangramento, hipotensão e necessidade contínua de administração de fluidos, caracteriza o chamado "Blood Vicious Cycle" (Ciclo Vicioso do Sangramento) que ainda desafia cirurgiões e intensivistas, devido à dificuldade de ser "quebrado" e de ser instituída uma reposição volêmica adequada, que garanta uma boa perfusão dos órgãos sem diluir excessivamente o sangue, causando mais hemorragia.
Para fins didáticos, discutiremos os principais fatores responsáveis pelo desenvolvimento da coagulopatia do trauma individualmente neste artigo:

\section{1- Hemodiluição}

O processo normal de coagulação requer um equilíbrio adequado nas concentrações dos diversos componentes envolvidos na hemostasia sanguínea. A administração de cristalóides e concentrado de hemácias na fase inicial do tratamento da perda sanguínea do politraumatizado resulta na diluição de fatores de coagulação, plaquetas e fibrinogênio. Estima-se que o volume de plaquetas presente no sangue de um adulto é apenas de $15 \mathrm{ml}$, e que a concentração de fibrinogênio disponível na circulação geralmente não ultrapassa 10 gramas, fazendo com que a atividade destes componentes esteja bastante sujeita a alterações durante esta administração de fluidos. ${ }^{9}$

A reposição volêmica inicial com cristalóides, durante o transporte de traumatizados pelas equipes de resgate, a administração adicional de fluidos, incluindo concentrado de hemácias pela equipe de trauma na emergência, assim como, o atraso na reposição de fatores de coagulação são os principais responsáveis 
pela hemodiluição encontrada na vítima de sangramento. Alguns estudos também apontam que o soro fisiológico e o ringer lactato agravam a lesão de reperfusão tecidual e aumentam a adesão leucocitária, e que o uso deles como fluido de restauração em trauma, pode piorar a acidose e a coagulopatia, e possivelmente aumentar a incidência de Síndrome da Angústia Respiratório do Adulto (SARA), Síndrome da Resposta Inflamatória Sistêmica (SRIS) e Falência de Múltiplos Órgãos (FMO). ${ }^{10}$

\section{2- Acidose}

Acidose é um dos mais importantes fatores que contribuem para a coagulopatia do trauma. A hipoperfusão dos tecidos durante o estado de choque e conseqüente metabolismo anaeróbico resulta em acidose metabólica láctica. Na condição de acidose, a atividade dos fatores de coagulação e plaquetas é diminuída. O fator VII ativado, por exemplo, tem sua função reduzida em torno de $90 \%$ quando o pH sanguíneo é reduzido de 7,4 para $7,0 .{ }^{11}$

O nível de lactato sanguíneo e a capacidade do fígado em normalizá-lo nas primeiras 24 horas do atendimento ao traumatizado em choque hemorrágico, foi relacionado com chances de sobrevivência de cerca de $100 \% .^{12,13}$

O uso de soro fisiológico também pode contribuir para esta condição, devido à acidose metabólica hiperclorêmica que se desenvolve com a administração agressiva deste tipo de fluido.

\section{3- Hipotermia}

Hipotermia é, sem dúvida, uma das grandes responsáveis pelo desenvolvimento da coagulopatia do trauma. Em vítimas de traumatismos, a queda da temperatura corporal central abaixo de $35^{\circ} \mathrm{C}$, é geralmente aceita para definição da condição de hipotermia. Acredita-se que a redução da temperatura afeta a cascata da coagulação através da restrição da atividade das enzimas envolvidas na função plaquetária e por meio de alterações endoteliais e no sistema fibrinolítico. ${ }^{13}$

Vários mecanismos são responsáveis pela queda da temperatura corporal do politraumatizado. Em regiões de climas mais frios ou durante meses de baixa temperatura e em setores de emergências e bloco cirúrgicos com sistemas de refrigeração, a perda de calor decorrente da convecção e radiação, assim como perdas evaporativas, em caso de traumatizados que permanecem com roupas molhadas por um período mais prolongado, são os principais responsáveis pela queda da temperatura nestes momentos inicias que seguem um acidente. $\mathrm{O}$ próprio estado de choque hemorrágico, onde existe uma diminuição no consumo de oxigênio ao nível celular, com conseqüente diminuição na produção de calor pelo metabolismo corporal é um outro fator que contribui para a hipotermia destes pacientes. A abertura das cavidades peritoneal e pleural, com exposição destas superfícies ao meio externo, durante procedimentos cirúrgicos, resulta também na perda de calor corporal. Um outro mecanismo importante que pode ser prevenido é a queda da temperatura decorrente da administração de fluidos endovenosos na temperatura ambiente ou refrigerados, não aquecidos até a temperatura corporal normal. ${ }^{6}$

Um estudo conduzido por Ferrara et al, que avalia a temperatura das vítimas de traumatismos, mostrou que a temperatura de $34^{\circ} \mathrm{C}$ ou inferior, estava presente em cerca de $80 \%$ dos pacientes que faleceram, enquanto apenas $36 \%$ dos sobreviventes, tiveram estes níveis de hipotermia, indicando que os níveis de temperatura nas vítimas de trauma estão associados com mortalidade. ${ }^{14}$

\section{3- DIAGNÓSTICO DA COAGULOPATIA DO TRAUMA}

O diagnóstico da coagulopatia do trauma é baseado nas alterações encontradas na avaliação clínica do doente, assim como nos dados dos exames laboratoriais. A evidência clínica da coagulopatia é a presença de sangramento contínuo de forma difusa, proveniente das áreas traumatizadas, de áreas do corpo cortadas ou dissecadas cirurgicamente, de pontos de inserção de cateteres e de mucosas.

Os exames de sangue mais utilizados para o diagnóstico desta condição são o tempo de protrombina (TP) e o tempo de tromboplastina parcial ativado (TTPa) ( $>1,5$ vezes os valores normais), a contagem de plaquetas $\left(<100 \times 10^{9} / 1\right)$ e o nível sanguíneo de fibrinogênio $(<1,0 \mathrm{~g} / \mathrm{l})$. Baseado nas alterações encontradas nestes exames existem várias definições e tentativas de se categorizar a gravidade da coagulopatia, publicadas recentemente na literatura mundial (Tabela II). ${ }^{13,15 / 20}$

\section{4- FATORES DE RISCO RELACIONADOS COM O DESENVOLVIMENTO DA COA- GULOPATIA}

Algumas condições, quando da apresentação inicial da vítima de sangramento, estão intimamente relacionados com o desenvolvimento de um estado 
Tabela II: Definições de coagulopatia no trauma na literatura recente

\begin{tabular}{|c|c|c|c|}
\hline Fonte & \multicolumn{3}{|l|}{ Parâmetros } \\
\hline Cosgriff et al., $1997^{13}$ & \multicolumn{3}{|c|}{ TP ou TTPa duas vezes o normal } \\
\hline Vaslef et al., $2002{ }^{15}$ & \multicolumn{3}{|c|}{$\mathrm{TP}>15$ " ou TTPa $>45$ " ou Fib $<1,0 \mathrm{~g} / 1$} \\
\hline Lynn et al., $2002{ }^{16}$ & \multicolumn{3}{|c|}{ Sangramento contínuo, difuso em cortes superficiais, cateter } \\
\hline Brohi et al., $2003{ }^{17}$ & \multicolumn{3}{|c|}{$\mathrm{TP}>18^{\prime \prime}$ ou $\mathrm{TTPa}>60 "$ ou $\quad$ TT $>15 ”$} \\
\hline MacLeod et al., $2003{ }^{18}$ & \multicolumn{3}{|c|}{ ТP $>14 "$ ou ТTPa $>34 "$} \\
\hline Dutton et al., $2004{ }^{19}$ & \multicolumn{3}{|c|}{ INR $>1.4$ ou $\mathrm{Plq}<100 \times 10^{9} / 1$ ou ambos } \\
\hline \multirow[t]{4}{*}{ Mayo et al., $2004^{20}$} & $\mathrm{TP} \geq 1,5 \times \mathrm{N}(0)$ & $1,5-2 \times N(1)$ & $>2 \times N(2)$ \\
\hline & $\mathrm{TTPa} \geq 1,5 \times \mathrm{N}(0)$ & $1,5-2 \times \mathrm{N}(1)$ & $>2 \times N(2)$ \\
\hline & $\mathrm{Plq} \geq 100 \times 10^{9} / \mathrm{l}(0)$ & $50-100 \times 10^{9} / 1(1)$ & $\leq 50 \times 10^{9} / 1(2)$ \\
\hline & Fib $>1,0 \mathrm{~g} / 1(0)$ & $0,5-1,0 \mathrm{~g} / \mathrm{l}(1)$ & $\leq 0,5 \mathrm{~g} / \mathrm{l}(2)$ \\
\hline
\end{tabular}

Total: 0 discreto; $1-3$ moderado; 2-8 grave.

TP - Tempo de protrombina; TTPa - Tempo de tromboplastina parcial ativado; Fib - Fibrinogênio; TT - Tempo de trombina; INR - International normalized ratio; Plq - Contagem de plaquetas; $\mathrm{N}$ - valor normal.

gravíssimo de coagulopatia. Estas condições incluem o nível de gravidade do trauma de acordo com o "Injury Severity Score - ISS" (> 25), o nível do pH sanguíneo $(<7.1)$, a temperatura corporal $\left(<34^{\circ} \mathrm{C}\right)$ e a pressão sistólica arterial $(<70 \mathrm{mmHg})$. Na nossa experiência, acreditamos que quando todos estes fatores estão presentes e o TTPa e o TP são pelo menos 2 vezes os valores normais, a chance de sobrevivência do traumatizado é mínima. Os valores de TP e TTPa por si só, também têm sido relacionado com o risco de morte nesta população de pacientes. ${ }^{18}$

\section{5- TRATAMENTO DA COAGULOPATIA DO TRAUMA}

Inquestionavelmente, a medida mais importante para prevenção da coagulopatia é o controle cirúrgico da hemorragia. As lesões vasculares com sangramento ativo devem ser reparadas imediatamente por meio da anastomose vascular ou ligadura definitiva de vasos sanguíneos. A compressão direta de áreas de sangramento facilita a coagulação de vasos lesados e previne perda adicional de sangue.

\section{1- Cirurgia de controle de danos - "Damage control surgery"}

A cirurgia do controle agudo do dano tem sido utilizada por mais de 20 anos e tem como objetivo combater o anteriormente mencionado "Ciclo Vicioso do
Sangramento". Em 1983, Stone e colaboradores descreveram a realização de cirurgias abreviadas, com o "packing" de órgãos com compressas (o preenchimento da cavidade abdominal com muitas compressas, no intuito de promover o tamponamento temporário de sangramentos provenientes de órgãos maciços ou outros vasos), com o objetivo de reverter o estado de coagulopatia, deixando o reparo definitivo dos órgãos lesados para um momento no qual o paciente se encontre mais estável, com temperatura e coagulação corrigidas. ${ }^{21} \mathrm{O}$ desenvolvimento de unidades de terapia intensivas especializadas no tratamento de traumatizados tem sido um fator determinante para a aplicação desta estratégia. Este modo de tratar vítimas de trauma inclui basicamente três etapas diferentes. Na primeira etapa, o cirurgião é o maior responsável tentando realizar procedimentos, o mais rapidamente possível, necessários apenas para conter sangramentos ativos, restabelecer o fluxo sanguíneo de órgãos e minimizar contaminações grosseiras. Com o objetivo de reduzir o tempo na sala de cirurgia, muitas vezes o abdômen ou tórax são parcialmente ou totalmente deixados abertos. Em seguida, o intensivista se encarrega de tratar a hipotermia, a acidose metabólica e a coagulopatia, otimizando o estado hemodinâmico e respiratório. Finalmente, entre 24 e 48 horas após a cirurgia inicial, o paciente é conduzido novamente ao centro cirúrgico com o objetivo de reparar definitivamente suas lesões. 
Esta estratégia é indicada para os grandes sangramentos, que requerem transfusão sanguínea maciça na emergência e no centro cirúrgico, em pacientes que se encontram acidóticos, hipotérmicos e com coagulopatia, onde a tentativa de reparar definitivamente as lesões consumiria tempo excessivo, colocando em risco a vida dos mesmos.

\section{2- Hipotensão permissiva - "Permissive hypotension"}

Hipotensão permissiva é uma outra forma de prevenir sangramentos adicionais que preconiza a condução inicial da vítima de traumatismo penetrante do tronco com certo nível de hipotensão e baseia-se no princípio de que a reposição volêmica maciça com fluidos produz altas pressões hidrostáticas nos vasos sanguíneos e, conseqüentemente, tendência a maior sangramento. A reposição de fluidos visa garantir mínima pressão arterial, suficiente para perfundir o cérebro e as artérias coronárias, adiando qualquer reposição adicional de fluidos até que o controle cirúrgico das lesões seja estabelecido. Esta forma de tratamento reduz a mortalidade em pacientes com lesões penetrantes do tronco. ${ }^{22}$ Entretanto, cautela deve existir na seleção dos pacientes, uma vez que a presença de trauma craniano associado é uma contra-indicação para aplicação da estratégia.

\section{3- Angiografia com embolização}

A utilização de angiografia para diagnóstico e tratamento de sangramentos ativos tem mostrado ser eficaz e rápida, particularmente nos casos de sangramentos arteriais associados a fraturas de bacia em pacientes com instabilidade hemodinâmica, apesar de fixação adequada da pelve. ${ }^{23}$

\section{4- Transfusão Sanguínea}

A transfusão inicial de concentrado de hemácias tem como função principal manter perfusão tecidual com oferta adequada de oxigênio ao nível celular. No entanto, como a anemia grave pode, individualmente, contribuir para o desenvolvimento de coagulopatia $^{24,25}$, recomenda-se manter níveis de hemoglobina acima de $8 \mathrm{~g} / \mathrm{dl}$ nesta fase de sangramento agudo, contudo lembrando que a medida da hemoglobina não é um bom preditor da velocidade de perda sangüínea nesta situação. ${ }^{26}$ Quando a condição clínica do traumatizado permite esperar cerca de 45 minutos, concentrado de hemácias é transfundido depois de classificação sanguínea e prova cruzada. Em situa- ções de maior emergência, sangue do tipo específico é transfundido. Em situações extremas, o tipo $\mathrm{O}$ negativo é utilizado para as mulheres em idade fértil e o tipo $\mathrm{O}$ positivo para qualquer outro caso.

A transfusão dos outros hemocomponentes (plaquetas, plasma e crioprecipitado) continua sendo a parte principal do tratamento da coagulopatia decorrente do sangramento maciço. Recomendações para o uso destes produtos para o tratamento da coagulopatia do trauma têm sido recentemente publicadas e foram sumarizadas na Tabela III. ${ }^{27}$

Transfusão de sangue e seus derivados trazem riscos e devem ser minimizadas ao máximo possível. Além do atualmente baixo risco de transmissão de vírus como o HIV (1:4.7 milhões), Hepatite B (1:82.000) e Hepatite C (1: 3.7 milhões), a prática da transfusão também carrega outros riscos como o desenvolvimento de sepses, SARA e FMO. Hipocalemia e hiperpotassemia também representam riscos da transfusão maciça. ${ }^{9}$

\section{5- Antifibrinolíticos}

Drogas como ácidos tranexâmico e amino-capróico têm sido usadas em cirurgias eletivas de grande porte para prevenir fibrinólise com redução do número de transfusões sanguínea. Entretanto, apesar de seu uso parecer muito atrativo para a coagulopatia do trauma, atualmente não existem dados de estudos em humanos que suportem a sua aplicação rotineira no trauma. ${ }^{28}$

\section{6- Agentes Locais de Coagulação}

Preparações para uso tópico contendo trombina e fibrinogênio têm sido utilizadas em cirurgias eletivas com resultados positivos. Estudos em animais sugerem que estes agentes talvez sejam eficientes em hemorragia do trauma. ${ }^{29}$

\section{6- LIMITAÇÕES DOS TRATAMENTOS ATUAIS}

Apesar de importantes avanços nas áreas de cirurgia, anestesia e terapia intensiva, no tocante ao tratamento das vítimas de traumatismos, as estratégias disponíveis ainda possuem uma série de limitações. A condição da vítima de traumatismo que sangra ativamente é peculiar no que concerne à necessidade de atuar de maneira rápida, instituindo-se medidas diagnósticas e terapêuticas muitas vezes quase que simultaneamente. Os testes laboratoriais de coa- 
Tabela III: Recomendações para transfusão de hemocomponentes para pacientes traumatizados em coagulopatia do Sunnybrook Health Sciences Centre, Canadá. ${ }^{9}$

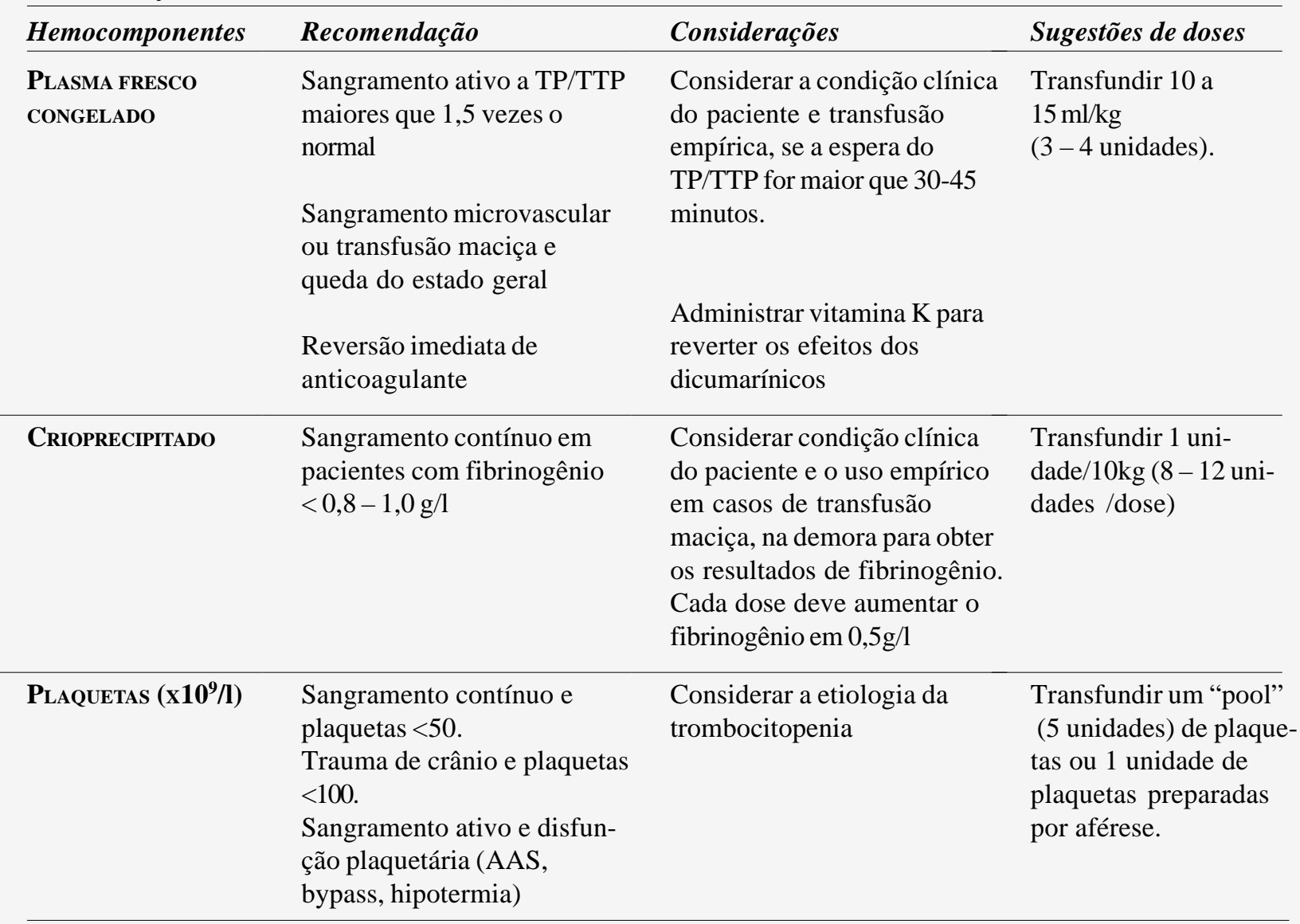

gulação que atualmente disponibilizamos avaliam partes isoladas do complexo processo de coagulação, e foram desenvolvidos para a monitarização de terapia com anti-coagulantes e os seus valores preditivos nunca foram validados no trauma. ${ }^{30}$ É importante lembrar também, que eles são realizados no laboratório, consumindo um tempo que pode ser determinante e com temperatura de $37^{\circ} \mathrm{C}$, o que, na maioria das vezes, não reflete a condição real do paciente.

Os protocolos de transfusão disponíveis atualmente para guiar a utilização de hemocomponentes são baseados apenas em estudos observacionais. ${ }^{31,32}$ As próprias recomendações do ATLS $^{\circledR}$ são extrapoladas de estudos em animais em circunstâncias controladas de sangramento, o que não representa, necessariamente, a condição nos pacientes traumatizados.

Em virtude das limitações encontradas nas estratégias atuais, o uso de sangue e seus derivados têm sido considerados inapropriados, e estudos retrospectivos têm comprovado o abuso na sua indicação. ${ }^{31,32}$

\section{7- PERSPECTIVAS DE NOVAS ESTRATÉGIAS PARA TRATAR A COAGULOPATIA DO TRAUMA}

\section{1- Teste de Coagulação}

Com a expectativa de uma melhor forma de avaliar, monitorar e orientar a correção da coagulopatia do trauma a tromboelastografia (TEG) parece ser potencialmente interessante. Apesar de ser uma tecnologia concebida há mais de 50 anos, o seu uso no trauma necessita de avaliação adequada. Em cirurgias de grande porte, como transplante hepático e cirurgia cardíaca, a TEG mostrou ter resultados positivos, reduzindo a quantidade de hemocomponentes transfun- 
didos. ${ }^{33-36}$ TEG pode avaliar todas as etapas do processo de coagulação, desde a atividade plaquetária até a fibrinólise, utilizando mínima quantidade de sangue e de forma rápida.

\section{2- Fator VII ativado Recombinante}

Produto aprovado para o tratamento de pacientes de hemofilia com sangramento, este fator recombinante de coagulação tem sido recentemente usado no tratamento de traumatizados com resultados positivos. O seu uso diminuiu sangramento e conseqüente transfusão de hemocomponentes em uma série de estudos retrospectivos e em um único estudo controlado e randomizado. ${ }^{37}$

Em um recente estudo canadense retrospectivo, o uso desta medicação para o tratamento de traumatizados com coagulopatia após transfusão sanguínea maciça, mostrou também ser eficaz na redução de mortalidade em 24 horas. ${ }^{8}$ Apesar de bastante promissora, esta droga precisa ainda ser melhor avaliada em estudos maiores, prospectivos e randomizados, que visem a mortalidade, a dose apropriada e os seus potenciais efeitos colaterais.

\section{3- “Damage Control” e Ressuscitação}

Com a idéia de abordar o desenvolvimento da coagulopatia nos sangramentos dos traumatizados, experiência com soldados americanos vitimados nos campos de batalha, aponta para um novo direcionamento na forma de transfundir. Cerca de $13 \%$ das transfusões em soldados americanos no Iraque, por exemplo, constituiu-se do uso de sangue total. ${ }^{38}$ Esta prática baseia-se na idéia da reposição não só de hemácias, mas também dos fatores de coagulação e plaquetas de uma forma muito precoce e simultânea, como que em antecipação à formação da coagulopatia. Esta estratégia precisa ser melhor avaliada através de estudos prospectivos e randomizados.

\section{8- CONCLUSÕES}

A coagulopatia que se desenvolve nos grandes sangramentos é a principal responsável pelos óbitos que ocorrem nas primeiras 24 horas do tratamento das vítimas de traumatismos. A terapêutica destes pacientes é complexa e muitas vezes desafiadora, necessitando um entendimento adequado da fisiopatologia da coagulopatia do trauma. Vários fatores contribuem para o seu desenvolvimento, notavelmente a hemodiluição, acidose e hipotermia. A principal medida para prevenção e tratamento desta condição é o controle cirúrgico imediato e de forma abreviada dos sangramentos. A correção da hipotermia e da acidose deve ser perseguida agressivamente, na unidade de terapia intensiva. $\mathrm{O}$ uso racional de sangue e hemocomponentes é fundamental na condução destes pacientes com o intuito de maximizar os efeitos da terapêutica e prevenir potenciais complicações da mesma. A arteriografia com embolização de vasos sangrantes tem sido um importante avanço na área. Felizmente, novas técnicas para diagnóstico e orientação da terapia transfusional continuam a ser desenvolvidas, assim como agentes sintéticos que têm o potencial de conter sangramentos e substituir componentes do sangue.

Nascimento B, Scarpelini S, Rizoli S. Trauma coagulopathy and transfusional therapy. Medicina (Ribeirão Preto) 2007; 40 (4): 509-17, oct./dec.

ABSTRACT: Trauma is one of the most important causes of deaths worldwide. Massive bleeding is the leading cause of trauma deaths in the first 24 hours following an injury. Major trauma patients frequently develop coagulopathy, which is related to huge bleedings, fluid therapy with crystalloids and red blood cells, acidosis and hypothermia. The control of this situation is a challenge to surgeons and intensivists. In addition, trauma victims are one of the most important consumers of blood components. Recently, the adequacy of this massive transfusion has been put into question, particularly due to the lack of well-developed and clinically-proved guidelines. The objective of this article is to provide an overview of the pathophysiology of the trauma coagulopathy, discussing current strategies available for its management and new developments in the field.

Keywords: Trauma. Massive Blood Loss. Coagulopathy. Blood Transfusion. 


\section{REFERENCES}

1 - Krug EG, Sharma GK, Lozano R. The global burden of injuries. Am J Public Health 2000;90:523-6.

2 - Brasil. Ministerio da Saude. Datasus. Informacao de Saude. Ministerio da Saude. Disponivel em URL: http://tabnet.datasus. gob.br/cgi/deftohtm.exe?idb2006/c04.def. [2007 jun 22].

3 - Projeto de Atenção Nacional ao Trauma. Sociedade Brasileira de Atendimento Integrado ao Traumatizado. disponivel em URL: http://www.paginet.com.br/sbait/projeto.html . [2007 jun 22].

4 - Sauaia A, Moore FA, Moore EE, Moser KS, Brennan RRN, Read RA, Pons PT. Epidemiology of trauma deaths: a reassessment. J Trauma 1995;38:185-93.

5 - Holcomb JB. Methods for improved hemorrhage control. Crit Care 2004;8 (Suppl 2):S57-S60.

6 - American College of Surgeons. Committee on Trauma. ATLS - Advanced Trauma Life Support for Doctors. Chicago: ACS; 1997.

7 - Como JJ, Dutton RP, Scalea TM, Edelman BB, Hess JR. Blood transfusion rates in the care of acute trauma. Transfusion. 2004;44:809-13.

8 - Rizoli SB, Nascimento B, Jr., Osman F et al. Recombinant activated coagulation factor VII and bleeding trauma patients. J Trauma. 2006;61:1419-25.

9 - Callum J.L., Pinkerson P.H. Bloody easy 2: blood transfusion, blood alternatives and transfusion reactions: a guide to transfusion medicine. Toronto: Savattuq Inc.; 2005.

10 - Coimbra R, Hoyt DB, Junger WG, Angle N, Wolf P, Loomis W et al. Hypertonic saline resuscitation decreases susceptibility to sepsis after hemorrhagic shock. J Trauma 1997;42:602-6.

11 - Meng ZH, Wolberg AS, Monroe DM, III, Hoffman M. The effect of temperature and $\mathrm{pH}$ on the activity of factor VIla: implications for the efficacy of high-dose factor VIla in hypothermic and acidotic patients. J Trauma 2003;55:886-91.

12 - Cotton BA, Guy JS, Morris JA, Jr., Abumrad NN. The cellular, metabolic, and systemic consequences of aggressive fluid resuscitation strategies2. Shock 2006;26:115-21.

13 - Cosgriff N, Moore EE, Sauaia A, Kenny-Moynihan M, Burch JM, Galloway B. Predicting life-threatening coagulopathy in the massively transfused trauma patient: hypothermia and acidoses revisited. J Trauma 1997;42:857-61.

14 - Ferrara A, MacArthur JD, Wright HK, Modlin IM, McMillen MA. Hypothermia and acidosis worsen coagulopathy in the patient requiring massive transfusion. Am J Surg 1990;160:515-8.

15 - Vaslef SN, Knudsen NW, Neligan PJ, Sebastian MW. Massive transfusion exceeding 50 units of blood products in trauma patients. J Trauma 2002;53:291-5.

16 - Lynn M, Jeroukhimov I, Klein Y, Martinowitz U. Updates in the management of severe coagulopathy in trauma patients. Intensive Care Med 2002;28 (Suppl 2):S241-7.

17 - Brohi K, Singh J, Heron M, Coats T. Acute traumatic coagulopathy. J Trauma 2003;54:1127-30.

18 - MacLeod JB, Lynn M, McKenney MG, Cohn SM, Murtha M. Early coagulopathy predicts mortality in trauma. J Trauma 2003; 55:39-44.

19 - Dutton RP, McCunn M, Hyder M, D’Angelo M, O'Connor J, Hess JR,Scalea TM. Factor VIla for correction of traumatic coagulopathy. J Trauma 2004;57:709-18.

20 - Mayo A, Misgav M, Kluger Y, Geenberg R, Pauzner D, KlausnerJ, Ben-Tal O. Recombinant activated factor VII (NovoSeven): addition to replacement therapy in acute, uncontrolled and life-threatening bleeding. Vox Sang 2004;87:34-40.
21 - Stone HH, Strom PR, Mullins RJ. Management of the major coagulopathy with onset during laparotomy. Ann Surg 1983; 197:532-5.

22 - Bickell WH, Wall MJ, Jr., Pepe PE, Martin RR, Ginger VF, Allen MK, Mattox KL. Immediate versus delayed fluid resuscitation for hypotensive patients with penetrating torso injuries. $\mathrm{N}$ Engl J Med 1994;331:1105-9.

23 - Lopez PP. Unstable pelvic fractures: the use of angiography in controlling arterial hemorrhage. J Trauma. 2007;62:S30-1.

$24-\mathrm{Ho} \mathrm{CH}$. The hemostatic effect of packed red cell transfusion in patients with anemia. Transfusion 1998;38:1011-4.

25 - Valeri CR, Cassidy G, Pivacek LE, Ragno G, Lieberthal W, Crowley JP et al. Anemia-induced increase in the bleeding time: implications for treatment of nonsurgical blood loss. Transfusion 2001;41:977-83.

26 - Stainsby D, MacLennan S, Thomas D, Isaac J, Hamilton PJ. Guidelines on the management of massive blood loss. $\mathrm{Br} \mathrm{J}$ Haematol. 2006;135:634-41.

27 - Tien H, Nascimento B, Jr., Callum J, Rizoli S. An approach to transfusion and hemorrhage in trauma: current perspectives on restrictive transfusion strategies. Can J Surg 2007; 50:202-9.

28 - Coats T, Roberts I, Shakur H. Antifibrinolytic drugs for acute traumatic injury. Cochrane Database Syst Rev 2004; CD004896.

29 - Holcomb JB, Pusateri AE, Harris RA, Reid TJ, Beall LD, Hess JR et al. Dry fibrin sealant dressings reduce blood loss, resuscitation volume, and improve survival in hypothermic coagulopathic swine with grade $\mathrm{V}$ liver injuries. J Trauma 1999; 47:233-40.

30 - Dzik WH. Predicting hemorrhage using preoperative coagulation screening assays. Curr Hematol Rep 2004;3:324-30.

31 - Dries DJ. Hypotensive resuscitation. Shock 1996;6:311-6.

32 - Garner AA, Bartolacci RA. Massive prehospital transfusion in multiple blunt trauma. Med J Aust 1999;170:23-5.

33 - Kang YG, Martin DJ, Marquez J, Lewis JH, Bontempo FA, Shaw BW Jr et al. Intraoperative changes in blood coagulation and thrombelastographic monitoring in liver transplantation. Anesth Analg 1985;64:888-96.

34 - Spiess BD, Gillies BS, Chandler W, Verrier E. Changes in transfusion therapy and reexploration rate after institution of a blood management program in cardiac surgical patients. J Cardiothorac Vasc Anesth 1995;9:168-73.

35 - Shore-Lesserson L, Manspeizer HE, DePerio M, Francis S, Vela-Cantos F, Ergin MA. Thromboelastography-guided transfusion algorithm reduces transfusions in complex cardiac surgery. Anesth Analg 1999;88:312-9.

36 - Nuttall GA, Garrity JA, Dearani JA, Abel MD, Schroeder DR, Mullany CJ. Risk factors for ischemic optic neuropathy after cardiopulmonary bypass: a matched case/control study. Anesth Analg 2001; 93:1410-6.

37 - Scarpelini S, Rizoli S. Recombinant factor VIla and the surgical patient. Curr Opin Crit Care 2006;12:351-6.

38 - Holcomb JB, Jenkins D, Rhee P, Johannigman J, Mahoney P, Mehta S, Cox E et al. Damage control resuscitation: directly addressing the early coagulopathy of trauma. J Trauma 2007; 62:307-10.

Recebido em 29/06/2007

Aprovado em 05/11/2007 